\title{
Assessing risk profiles for Salmonella serotypes in breeding pig operations in Portugal using a Bayesian hierarchical model
}

\author{
Carla Correia-Gomes ${ }^{1,2^{*}}$, Theodoros Economou ${ }^{3}$, Denisa Mendonça ${ }^{1,2}$, Madalena Vieira-Pinto ${ }^{4}$
} and João Niza-Ribeiro ${ }^{1,2}$

\begin{abstract}
Background: The EU Regulation No 2160/2003 imposes a reduction in the prevalence of Salmonella in pigs. The efficiency of control programmes for Salmonella in pigs, reported among the EU Member States, varies and definitive eradication seems very difficult. Control measures currently recommended for Salmonella are not serotype-specific. Is it possible that the risk factors for different Salmonella serotypes are different? The aim of this study was to investigate potential risk factors for two groups of Salmonella sp serotypes using pen faecal samples from breeding pig holdings representative of the Portuguese pig sector.
\end{abstract}

Methods: The data used come from the Baseline Survey for the Prevalence of Salmonella in breeding pigs in Portugal. A total of 1670 pen faecal samples from 167 herds were tested, and 170 samples were positive for Salmonella. The presence of Salmonella in each sample (outcome variable) was classified in three categories: i) no Salmonella, ii) Salmonella Typhimurium or S. Typhimurium-like strains with the antigenic formula: 1,4,5,12:i:-, and iii) other serotypes. Along with the sample collection, a questionnaire concerning herd management and potential risk factors was utilised. The data have a "natural" hierarchical structure so a categorical multilevel analysis of the dataset was carried out using a Bayesian hierarchical model. The model was estimated using Markov Chain Monte Carlo methods, implemented in the software WinBUGS.

Results: The significant associations found (when compared to category "no Salmonella"), for category "serotype Typhimurium or $S$. Typhimurium-like strains with the antigenic formula: 1,4,5,12:i:-" were: age of breeding sows, size of the herd, number of pigs/pen and source of semen. For the category "other serotypes" the significant associations found were: control of rodents, region of the country, source of semen, breeding sector room and source of feed.

Conclusions: The risk factors significantly associated with Salmonella shedding from the category "serotype Typhimurium or serotype 1,4,5,12:i:-" were more related to animal factors, whereas those associated with "other serotypes" were more related to environmental factors. Our findings suggest that different control measures could be used to control different Salmonella serotypes in breeding pigs.

\footnotetext{
* Correspondence: carla.gomes@sruc.ac.uk

${ }^{1}$ Instituto de Ciências Biomédicas Abel Salazar - Universidade do Porto

(ICBAS-UP), Population Studies Department, Largo Prof. Abel Salazar, Porto 2, 4099-003, PORTUGAL

${ }^{2}$ Instituto de Saúde Pública da Universidade do Porto (ISPUP), Rua das Taipas, Porto 135, 4050-600, PORTUGAL

Full list of author information is available at the end of the article
} 


\section{Background}

Salmonella has been one of the major causes of foodborne disease in the European Union (EU) in the past years [1]. A considerable proportion of human cases are related to pork products [2]. The EU approved legislation (EU Regulation No 2160/2003) imposes a reduction on the prevalence of this agent in food production animals, such as pigs. To set the target for this reduction per country, baseline surveys were carried out in the EU to estimate the prevalence of Salmonella sp. in some food production animals. The objective of the surveys was to obtain comparable data for all Member States (MS) through a harmonized approach. These studies showed that the prevalence of Salmonella in holdings with breeding pigs was $31.8 \%$ (28.7\% for breeding holdings and $33.3 \%$ for production holdings) [3] and also that there are different profiles in terms of serotype prevalence among different countries. In Portugal for instance, $9.1 \%$ of the breeding holdings were positive to Salmonella Typhimurium and $33.3 \%$ were positive to other serotypes than Typhimurium and Derby, while in Ireland these numbers were $17.5 \%$ for both cases [3]. Another important issue is that control programmes already being carried out in several MS have different efficiencies and so far, none seems to be able to reduce the level of Salmonella sp. to reach an eradication stage [4]. Control programmes should target all serotypes of Salmonella sp., since all of them have the potential to be pathogenic for humans. To improve the efficiency of control programmes, potential differences in serotypes prevalence which allows for differences in risk factors between serotypes should be taken in consideration. Some of the known risk factors in the literature are linked to: 1) biosecurity measures [5] especially those aimed at potential biological vectors (rodents) [6-8], hand, equipment and facility hygiene [9] and also purchase of animals from different suppliers [9]; 2) herd management - such as herd size [10], batch production system [11], housing - type of floor (partial slatted floor) [12,13] and type of pen [9]; 3) feeding practices such as dry feed [14], source of feed [15] and adding organic acids to feed [11]; 4) health disorders such as use of antibiotics $[16,17]$, parasite infestations $[18,19]$, and health status of the herd [11] among others. However, none of the aforementioned studies have taken into consideration whether risk factors differ between serotypes. To the best of our knowledge only one study compared the differences between risk factors for Salmonella serotypes with or without antimicrobial resistance [20]. The data for this paper were collected by the Portuguese Veterinary Authority (PVA) when the Baseline Survey on the Prevalence of Salmonella in breeding pigs was conducted in Portugal. The aim was to search for potential risk factors for shedding from two different groups of
Salmonella serotypes using pen faecal samples from herds with breeding pig representative of Portuguese reality. The two groups were Salmonella Typhimurium including $S$. Typhimurium-like strains with the antigenic formula: 1,4,5,12:i:-, and other serotypes.

\section{Methods}

\section{Herd selection}

The objectives, the sampling frame, the diagnostic testing methods as well as the collection and reporting of data, and the timelines of the Baseline Survey on the Prevalence of Salmonella in breeding pigs were specified in the Commission Decision 2008/55/EC. The target population are holdings that constitute at least $80 \%$ of the breeding pig population in the Member State.

The sample size was calculated by the PVA and considered the number of swine herds existing in April of 2007, stratified by Region. The sampling frame consisted of 4522 herds, with 204,584 breeding pigs and 1,827,533 pigs in total. The herd inclusion criteria for entering the sampling frame were: to have at least 50 breeding pigs, either for breeding or production purposes. The pig population included in the sampling frame represented $87 \%$ of the total registered pig population in Portugal in 2007.The sample size was calculated using the sampling criteria specified in the Commission Decision 2008/55/ EC Annex I - expected herd prevalence of 50\%, desired confidence level of $95 \%$, accuracy of $7.5 \%$ and then apply a finite population correction factor, with an increase of $10 \%$ for each group (breeding and production holdings) in case of non-response. A sample of 174 swine herds was randomly selected using probability proportional to the number of herds among the regions in Portugal.

\section{Pen selection}

In each herd only the pens with breeding pigs over six months of age were randomly selected. The breeding pigs that have been recently introduced into the herd and were in quarantine were not included in the survey. In each selected herd, faecal samples from 10 pens were taken representing a $95 \%$ probability of detecting at least one positive sample if the true prevalence of infected pigs in the population was $10 \%$ [21]. The number of pens sampled per breeding room in each herd was allocated proportionally according to the number of breeding pigs in the different stages of production. The age categories in the sampling were not predetermined. The specification was that at least 10 individual breeding pigs should be included in each pooled pen faecal sample otherwise no sample was collected.

\section{Faecal samples collection}

The faecal samples were collected and pooled together by the herd veterinary assistant and then sent to 
laboratory for detection of Salmonella. The material consisted of freshly voided faeces. Each pooled sample should weigh at least $25 \mathrm{~g}$ and two approaches were employed to collect these pooled faeces samples: 1) where there was an accumulation of mixed faeces within an area of a pen or yard, a large swab was used to pass through the faecal mass, ensuring that at least $25 \mathrm{~g}$ of mixed material was collected; 2) where there was no such accumulation (e.g. field, large yard, farrowing house, pens or other accommodation with low numbers of pigs per group) then individual pinches were selected from individual fresh faecal masses or places with a minimum of 10 individuals contributing to the final volume of at least $25 \mathrm{~g}$. The sites from which these pinches

Table 1 Herd variables assessed by the questionnaire and distribution of the pen samples by the categories of the outcome variable

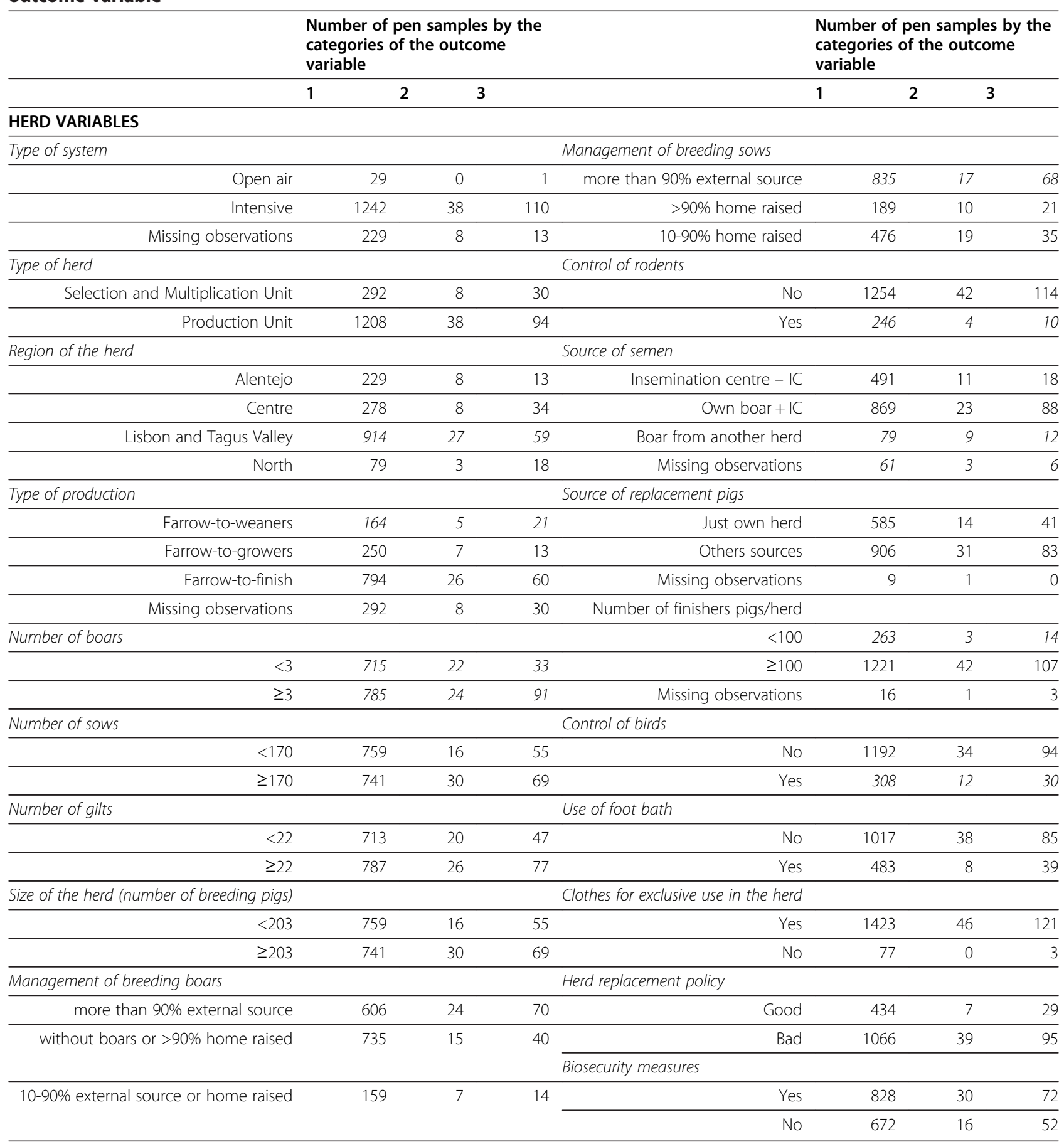


were collected were distributed in a representative manner across the area concerned. In approach 1) at least 10 individual pigs contributed to each sample taken, otherwise approach 2) was applied (Commission Decision 2008/55/EC).

\section{Salmonella isolation}

At the laboratory, the isolation of Salmonella was done using the method described by Annex D of ISO 6579. The Salmonella strains isolated in the positive pen faecal samples were serotyped in the National Reference Laboratory for Salmonella according to Kaulfmann-White scheme. The sensitivity of cultured pooled faecal samples according to the described method varied around $80 \%$ and the specificity is $100 \%[22,23]$.

\section{Data collection}

A questionnaire was used to collect information about the herd management and potential risk factors for Salmonella sp. shedding. This was filled by the herd veterinary who also collected the faecal samples (both tasks were conducted the same day). The questionnaire was designed by the PVA following the guidelines of Commission Decision 2008/55/EC. To minimize the bias that could be introduced by having different people collecting the data, the following procedures were taken: the majority of the questions were closed, the questionnaire had clear filling instructions attached and clarification meetings were held between the PVA and the field veterinarians before the sample collection took place. All the variables in the questionnaire are shown in Tables 1 and 2.

Table 2 Pen variables assessed by the questionnaire and distribution of the pen samples by the categories of the outcome variable

\begin{tabular}{|c|c|c|c|c|c|c|c|c|}
\hline & \multicolumn{4}{|c|}{$\begin{array}{l}\text { Number of pen samples } \\
\text { by the categories of the } \\
\text { outcome variable }\end{array}$} & & \multicolumn{3}{|c|}{$\begin{array}{l}\text { Number of pen samples } \\
\text { by the categories of the } \\
\text { outcome variable }\end{array}$} \\
\hline & 1 & 2 & 3 & & & 1 & 2 & 3 \\
\hline \multicolumn{9}{|l|}{ PEN VARIABLES } \\
\hline The pen has direct access to outside & & & & & Sanitary gap before breeders entering & & & \\
\hline No & 1146 & 30 & 30 & 92 & No & 626 & 19 & 44 \\
\hline Yes & 354 & 16 & 16 & 32 & Yes & 874 & 27 & 80 \\
\hline Individual pen & & & & & Feed & & & \\
\hline No & 1194 & 41 & 41 & 98 & Dry pellet & 229 & 7 & 27 \\
\hline Yes & 306 & & 5 & 24 & Dry non pellet & 1230 & 36 & 97 \\
\hline Missing observations & 0 & & 0 & 2 & Wet & 41 & 3 & $c$ \\
\hline Diarrhoea in the last 3 months & & & & & Floor & & & \\
\hline No & 1445 & 45 & 45 & 118 & Fully slatted & 139 & 5 & 10 \\
\hline Yes & 33 & & 1 & 2 & Others & 1361 & 41 & 112 \\
\hline Missing observations & 22 & & 0 & 4 & Source of feed & & & \\
\hline Age of the breeding sows & & & & & Exclusively own & 199 & 8 & $\varepsilon$ \\
\hline Only gilts or gilts and others & 874 & 38 & 38 & 73 & Bought + Mixture & 1301 & 38 & 116 \\
\hline Without gilts & 626 & & 8 & 51 & Potential Salmonella control substances added to water & & & \\
\hline Sex of the breeding pigs & & & & & No & 1291 & 38 & 11 \\
\hline Only females & 1430 & 44 & 44 & 114 & Yes & 209 & 8 & 13 \\
\hline Males and females & 70 & 2 & 10 & & Use of antibiotics in the last 4 weeks in breeders & & & \\
\hline Breeding sector & & & & & No & 1229 & 45 & 103 \\
\hline Mating room & 210 & 10 & 10 & 21 & Yes & 271 & 1 & 2 \\
\hline Gestation room & 789 & 26 & 26 & 62 & Way how was collected the sample & & & \\
\hline Mixture of room & 58 & & 1 & 14 & Compose sample & 121 & 1 & 11 \\
\hline Farrowing room & 390 & & 7 & 22 & Swab & 1379 & 45 & 113 \\
\hline Replacement breeders & 44 & 2 & 5 & & Number of pigs in the pen & & & \\
\hline & & & & & $=10$ & 1284 & 34 & 92 \\
\hline & & & & & $>10$ & 216 & 12 & 30 \\
\hline
\end{tabular}

Legend:1 (no Salmonella), 2 (serotype Typhimurium or serotype 1,4,5,12:i:-), 3 (other serotypes). 


\section{Data analysis}

From the information gathered in the questionnaires, two new binary variables were created. The first variable groups the questions regarding management of replacing breeding pigs and their source, and was codified as Good if more than $90 \%$ of the breeding sows and boars were homebred (also included herds with no boars) and if the semen was not from another herd otherwise it was codified as Bad. The second variable combines the questions about biosecurity measures and was codified as Yes when controls for rodents and birds were implemented, and also if herds had provisions for foot bathing and clothe changing before entering the herd and No otherwise. The variables and their categories were recoded or aggregated to fewer categories as necessary to avoid sparse data problems as shown in Tables 1 and 2 .

The continuous variables were transformed into categorical using the median values as the cut-off points defining the categories. Their summary statistics are shown in Table 3.

Because of the low number of cases per serotype (Table 4), individual analysis of each Salmonella serotype was prohibitive. Therefore the outcome variable was the isolation of Salmonella in each sample and was classified in three categories: i) no Salmonella, ii) serotype Typhimurium and $S$. Typhimurium-like strains with the antigenic formula: 1,4,5,12:i:-, and iii) other serotypes. For the calculation of apparent herd prevalence, a herd was considered positive if it had at least one positive pen faecal sample. The percentage of positive Salmonella sp. pen faecal samples was $27 \%$ Salmonella Typhimurium or $S$. Typhimurium-like strains with the antigenic formula: 1,4,5,12:i:-, and $73 \%$ other serotypes.

The data have a "natural" multilevel structure: pen faecal samples (first level) nested in herds (second level) and were analysed using a Bayesian hierarchical model with a categorical response variable (three categories). Monte Carlo Markov Chain (MCMC) was used for estimation and this was implemented in the freely available software WinBUGS (BUGS project, http://www.mrc-bsu. cam.ac.uk/bugs/winbugs/). Hierarchical models are naturally handled in the Bayesian framework because of the conditional independence assumed between each level in the hierarchy. In conjunction with the open-source software WinBUGS, this provides a general framework for implementing hierarchical models in similar applications.

Random effects were included at the herd level to account for the fact that the observations are 'nested' in herds. Treating the herd effect as random, also allows for the fact that the number of herds here (167) is a sample of all existing herds. All prior distributions were chosen to be as uninformative as possible. A more detailed description of the model is given in Additional file 1 .

To decide which variables should be included in this multivariable model, an exploratory analysis was performed by fitting univariable models and considering as candidates for the multivariable model, all variables significant at the 0.15 significance level. Associations between the explanatory variables were tested using a chisquare test and if a significant association $(\mathrm{p}<0.05)$ was found, only the variables with more biological justification were allowed to enter the model.

The final multivariable model was built using a forward selection process until all variables with a significant $95 \%$ credible interval were included. The significance level was set at 0.05 .

The model ran long enough with sufficient burn-in (5000 iterations) to ensure convergence to the posterior distribution of the parameters. Convergence was assessed by visual inspection of the means in time-series plots but also more formally using the Raftery and Lewis, and the Gelman-Rubin R-hat diagnostics [24,25]. R-hat should be arbitrarily close to 1 for convergence. The chains were thinned by only collecting 1 in 10 consecutive samples and this eliminated autocorrelation in posterior samples (using the CODA package [26] in R). Mixing in the chains was assessed by comparing the MC (Markov Chain) error with the standard deviation, for each parameter. Ideally, the MC error should be less than $5 \%$ of the standard deviations for good mixing [27] and this was true for all parameters here. Two MCMC chains ran with dispersed initial values which is good practice to ensure convergence and mixing. WinBUGS code for implementing the model is given in Additional file 2.

The presence of confounding was investigated by analysing the correlation matrix of the joint posterior

Table 3 Distribution of the continuous variables (at herd and pen level)

\begin{tabular}{|c|c|c|c|c|c|c|c|}
\hline Variable & Mean & Standard deviation & Minimum & Percentile 25 & Median & Percentile 75 & Maximum \\
\hline Number of boars & 3.9 & 3.9 & 0 & 2 & 3 & 4 & 28 \\
\hline Number of sows & 226.5 & 192.9 & 8 & 98 & 170 & 300 & 1077 \\
\hline Number of gilts & 34.0 & 38.3 & 0 & 12 & 22 & 40 & 300 \\
\hline Size of the herd (number of breeding pigs) & 265.0 & 216.9 & 41 & 109 & 203 & 355 & 1214 \\
\hline Number of pigs per pen* & 11.6 & 8.0 & 10 & 10 & 10 & 10 & 130 \\
\hline
\end{tabular}

* 258 pens had more that 10 pigs per pen. 
Table 4 Percentage of serotypes isolated in the study

\begin{tabular}{|c|c|c|c|c|c|c|}
\hline \multirow[t]{2}{*}{ Serotype } & \multicolumn{3}{|c|}{ Percentage of isolates $(n)$} & \multicolumn{3}{|c|}{$\begin{array}{l}\text { Percentage of herds that have at least one pen sample positive to } \\
\text { the serotype (n) }\end{array}$} \\
\hline & Breeding holdings & Production holding & All Holdings & Breeding holdings & Production holding & All holdings \\
\hline Typhimurium & $15.8(6)$ & $25(33)$ & $23(39)$ & $13.6(3)$ & $25.6(20)$ & $13.2(23)$ \\
\hline Rissen & $18.4(7)$ & $19.7(26)$ & $19(35)$ & $22.7(5)$ & $19.2(15)$ & $12.0(20)$ \\
\hline London & $21(8)$ & $13.6(18)$ & $15(26)$ & $13.6(3)$ & $11.5(9)$ & $7.2(12)$ \\
\hline Derby & $15.8(6)$ & $9.1(12)$ & $11(18)$ & $13.6(3)$ & $8.9(7)$ & $6.0(10)$ \\
\hline Give & $13.1(5)$ & $5.3(7)$ & $7(12)$ & $9.1(2)$ & $5.1(4)$ & $4.0(6)$ \\
\hline Brandenburg & $0(0)$ & $6.1(8)$ & $5(8)$ & $0(0)$ & $2.6(2)$ & $1.8(2)$ \\
\hline 1,3,19:-:- & $2.6(1)$ & $4.5(6)$ & $4(7)$ & $4.5(1)$ & $6.4(5)$ & $3.6(6)$ \\
\hline 1,4,5,12:i:- & $5.3(2)$ & $3.8(5)$ & $4(7)$ & $9.1(2)$ & $3.8(3)$ & $3.0(5)$ \\
\hline Bovismorbificans & $0(0)$ & $3(4)$ & $2(4)$ & $0(0)$ & $2.6(2)$ & $1.2(2)$ \\
\hline Gloucester & $0(0)$ & $2.3(3)$ & $2(3)$ & $0(0)$ & $2.6(2)$ & $1.2(2)$ \\
\hline Muenchen & $2.6(1)$ & $2.3(3)$ & $2(4)$ & $4.5(1)$ & $3.8(3)$ & $2.4(4)$ \\
\hline Anatum & $0(0)$ & $1.5(2)$ & $1(2)$ & $0(0)$ & $2.6(2)$ & $1.2(2)$ \\
\hline Bredeney & $0(0)$ & $0.8(1)$ & $1(1)$ & $0(0)$ & $1.3(1)$ & $0.6(1)$ \\
\hline Goldcoast & $0(0)$ & $1.5(2)$ & $1(2)$ & $0(0)$ & $1.3(1)$ & $0.6(1)$ \\
\hline Livingstone & $2.6(1)$ & $0(0)$ & $1(1)$ & $4.5(1)$ & $0(0)$ & $0.6(1)$ \\
\hline Mbandaka & $2.6(1)$ & $0.8(1)$ & $1(2)$ & $4.5(1)$ & $1.3(1)$ & $1.2(2)$ \\
\hline Senftenberg & $0(0)$ & $0.8(1)$ & $1(1)$ & $0(0)$ & $1.3(1)$ & $0.6(1)$ \\
\hline
\end{tabular}

distribution for all model parameters but especially the slope parameters. Correlation values higher than 0.5 where takes to indicate significant correlation.

Posterior predictive simulation was used for model checking as described by Gilks et al.[28]. This technique is effectively testing whether the observed data are extreme in relation to the predictive distribution (fitted model). Model deviance was the measure adopted for comparison. The technique involves the estimation of a p-value which should not be extreme (close to 0 or 1 ) for good model fit.

\section{Results}

A total 167 herds (33 breeding and 134 production holdings) responded to the questionnaire and were tested: 76 herds were positive to Salmonella sp. (apparent prevalence of $45.5 \%$, CI: $37.9 \%$ - 53.1\%). Of these, 15 breeding holdings (apparent prevalence of $45.5 \%, \mathrm{CI}: 28.5 \%$ $62.4 \%$ ), and 61 production holdings (apparent prevalence of $45.5 \%$, CI: $37.1 \%-53.9 \%$ ) were positive to Salmonella sp. Among the 1670 faecal samples collected, 170 were positive $(10.1 \%)$ and seventeen different serotypes were found (Table 4). There was no simultaneous occurrence of the two groups of serotypes in any of the positive samples. Salmonella Typhimurium was found in $23 \%$ of the positive isolates $(15.8 \%$ in breeding and $25 \%$ in production holdings), followed by Salmonella Rissen (19\%) (Table 4). The proportion of the different serotypes by type of holding is detailed in Table 4. Considering the distribution of serotypes groups through the herds, it was observed that $13.8 \%$ of the herds had at least one sample positive to serotype Typhimurium or $S$. Typhimurium-like strains with the antigenic formula: $1,4,5,12: \mathrm{i}:-$, and $31.7 \%$ of the herds had at least one positive sample to other serotypes. A significant association was found between number of sows and number of breeding pigs and for this reason it was decided that only the number of breeding pigs should enter the multivariable model.

Several management practices linked to herd and pen were assessed (Tables 1 and 2). The variables - region of the herd, size of the herd, source of semen, rodents control, number of pigs per pen, age of breeding sows, breeding sector room, source of feed and use of antibiotics - were selected to enter the multivariable model. Table 5 shows the final multivariable multilevel model results. The results were converted to odds ratio (OR) and the respective $95 \%$ credible intervals (OR CrI) were calculated. The posterior median was used to estimate point values of each OR, because unlike the mean, this is less affected by asymmetric distributions. Posterior distributions of all OR are highly asymmetric since they are based on the exponentiation of posteriors of the slope parameters. The convergence of MCMC calculations was considered acceptable with R-hat values of all parameters being less than 1.001. Different starting values did not affect the final results. None of the between-parameter correlations was larger than 0.5 in magnitude while the majority was less than 0.1 implying no influential confounding in any of the variables. 
Table 5 Posterior results for the final multivariable categorical multilevel model for the risk factors (Salmonella negative as reference group)

\begin{tabular}{|c|c|c|c|c|c|c|c|c|}
\hline \multirow[t]{2}{*}{ Variable } & \multicolumn{4}{|c|}{ Typhimurium or $1,4,5,12: \mathrm{i}:-$} & \multicolumn{4}{|c|}{ Other serotypes } \\
\hline & Coefficient & SD & OR & $95 \%$ OR Crl & Coefficient & SD & OR & $95 \%$ OR Crl \\
\hline \multicolumn{9}{|l|}{ HERD } \\
\hline \multicolumn{9}{|l|}{ Region of the herd } \\
\hline Alentejo & 0 & & 1.0 & & 0 & & 1.0 & \\
\hline Centre & -1.3 & 1.5 & 0.28 & $0.01-4.30$ & 1.5 & 0.7 & 4.57 & $1.33-17.57$ \\
\hline Lisbon and Tagus Valley & -0.5 & 1.1 & 0.62 & $0.07-5.05$ & 0.9 & 0.6 & 2.56 & $0.86-8.36$ \\
\hline North & -0.1 & 1.7 & 0.88 & $0.03-24.31$ & 2.6 & 0.8 & 12.9 & $2.97-64.33$ \\
\hline \multicolumn{9}{|l|}{ Size of the herd: (number of breeding pigs) } \\
\hline$<203$ & 0 & & 1.0 & & 0 & & 1.0 & \\
\hline$\geq 203$ & 1.9 & 0.9 & 7.04 & $1.46-60.04$ & 0.5 & 0.4 & 1.65 & $0.83-3.44$ \\
\hline \multicolumn{9}{|l|}{ Source of semen } \\
\hline Insemination centre - IC & 0 & & 1.0 & & 0 & & 1.0 & \\
\hline Own boar + IC & 0.4 & 0.8 & 1.45 & $0.24-7.77$ & 1.1 & 0.4 & 2.91 & $1.35-6.83$ \\
\hline Boar from another herd & 3.7 & 1.6 & 41.22 & $2.46-1392.7$ & 1.4 & 0.8 & 4.18 & $0.94-19.30$ \\
\hline \multicolumn{9}{|l|}{ Control of rodents } \\
\hline No & 0 & & 1.0 & & 0 & & 1.0 & \\
\hline Yes & -2.2 & 1.8 & 0.11 & $0.002-1.85$ & -2.0 & 0.7 & 0.13 & $0.03-0.45$ \\
\hline \multicolumn{9}{|l|}{ PEN } \\
\hline \multicolumn{9}{|l|}{ Number of pigs/pen } \\
\hline$=10$ & 0 & & 1.0 & & 0 & & 1.0 & \\
\hline$>10$ & 1.4 & 0.7 & 4.06 & $1.03-19.73$ & 0.6 & 0.4 & 1.82 & $0.88-3.79$ \\
\hline \multicolumn{9}{|l|}{ Age of the breeding sows } \\
\hline Only gilts or gilts and others & 0 & & 1.0 & & 0 & & 1.0 & \\
\hline Without gilts & -1.8 & 0.8 & 0.17 & $0.03-0.65$ & 0.2 & 0.3 & 1.24 & $0.68-2.24$ \\
\hline \multicolumn{9}{|l|}{ Breeding sector room } \\
\hline Mating & 0 & & 1.0 & & 0 & & 1.0 & \\
\hline Gestation & 0.1 & 0.5 & 1.11 & $0.44-3.10$ & -0.2 & 0.3 & 0.81 & $0.45-1.52$ \\
\hline Mixture of animals of different sectors & 0.2 & 1.7 & 1.17 & $0.03-24.80$ & 0.8 & 0.7 & 2.14 & $0.54-7.78$ \\
\hline Farrowing & -1.0 & 0.6 & 0.36 & $0.10-1.22$ & -1.0 & 0.4 & 0.38 & $0.17-0.80$ \\
\hline Replacement breeders & -0.9 & 1.1 & 0.40 & $0.04-2.72$ & 0.1 & 0.7 & 1.15 & $0.29-3.88$ \\
\hline \multicolumn{9}{|l|}{ Source of feed } \\
\hline Exclusively own & 0 & & 1.0 & & 0 & & 1.0 & \\
\hline Not exclusively own & 0.5 & 1.1 & 1.63 & $0.18-17.62$ & 2.0 & 0.7 & 7.29 & $2.25-29.46$ \\
\hline Herd random effect variance & 5.8 & 0.66 & & & 1.4 & 0.24 & & \\
\hline
\end{tabular}

Legend: SD - standard deviation, OR - odds ratio, Crl - credible interval, in bold the significant OR for a 95\%Crl.

Significance of the herd random effects is tested by looking at whether the variance estimates $\left(1 / \tau_{1}, 1 / \tau_{2}\right)$ are non-zero. Estimates of $1 / \tau_{1}$ and $1 / \tau_{2}$ are arbitrarily away from zero (5.8 and 1.4 respectively) and their standard errors are relatively small, indicating that both estimates are different from zero (see Table 5). The model fit was reasonably accurate with a p-value of 0.21 which means no significant differences between replicated and observed data.

It can be seen from the analysis of Table 5 that there are different risk profiles for the two Salmonella serotype categories when compared to category "no
Salmonella". This is an important finding and suggests that the risk factors may be different between the categories of serotypes defined in this study. For category "Typhimurium or S. Typhimurium-like strains with the antigenic formula: 1,4,5,12:i:- associations with significant change in risk were: 1) size of the herd: herds with 203 and more breeding pigs are at higher risk of infection, 2) the source of semen: purchase of boars from other herds increase the risk of infection, 3) number of pigs per pen: pens with more than 10 animals per pen have increased risk of infection, and 4) the age of the sows: pens without gilts have a decreased risk of 
infection. For the category "other serotypes" the significant risk associations were: 1) region of the herd: herds in the Centre and North Region have a higher risk of infection, 2) the source of semen: the use of own boar increased the herds' risk of infection, 3) control of rodents had a significant effect in reducing the risk at the herd level, 4) feed source: using feed from external sources, i.e., not exclusively from the farm increased the risk of infection, and 5) breeding sector: the farrowing sector had a lower risk of infection than the mating sector.

\section{Discussion}

This study investigated risk factors for Salmonella shedding for two different groups of serotypes using pen faecal samples from herds with breeding pigs adequately representing the Portuguese pig industry.

\section{The outcome variable}

The different serotypes of Salmonella $s p$. were divided in two groups because serotype Typhimurium is a serotype with a recognized difficult control [29] and is also the cause of many human cases of food-borne disease linked to pork meat. Serotype Typhimurium-like strains with the antigenic formula: 1,4,5,12:i:-was included in the group of serotype Typhimurium because of the genetic similarity, the similar virulence and the antimicrobial resistance characteristics existing between the two serotypes [30]. The use of composite samples increases the overall sensitivity of detection of infected pens [31] strengthening the confidence on the accuracy of our response variable. The increased sensitivity of the use of pooled faecal samples was shown by the analysis of the Baseline Survey results [32] which demonstrated that this pooled sampling process detected approximately $80 \%$ of the true Salmonella positive herds, and that with 10 pooled faecal samples it is possible to detect at least one positive sample in a pig herd when the animal level prevalence is at least $20 \%$, with $95 \%$ certainty [31].

\section{The model}

It was anticipated that the hierarchical structure of the data from our sample could influence the outcome of the analysis. Therefore the statistical approach was chosen to take into consideration the multilevel structure of data from our sample where the pen faecal samples (level 1) are nested in herds (level 2). Some important remarks concerning the statistical approach deserve to be highlighted: the model implemented here showed a good fit, despite the fact there was little information to update the prior distributions. The methodology proposed could offer a general modelling approach to researchers who want to incorporate expert knowledge in the specification of the priors or for those who wish to restrict the priors accordingly to account for lack of information in the response variable which was not the case in this study. Lastly, both WinBUGS and R, are freely available software which is particularly appealing for the purpose of presenting the methodology here as a general modelling tool.

\section{Risk factors for Salmonella Typhimurium and Typhimurium-like strains with the antigenic formula: 1,4,5,12:i:- infection}

It can be seen from the analysis of Table 5 that there are different risk profiles for the two categories of Salmonella, validating our initial hypothesis that the risk factors could vary between the two categories of serotypes studied.

In category "Typhimurium or 1,4,5,12:1:-" the size of the herd (the number of breeding pigs being equal or greater than 203) was considered a risk factor. A similar association was found for Salmonella sp. in finishers [10] and also in the breeding pigs [32]. A reason for this is that in bigger herds, the risk of transmission is higher given a higher number of "infectious" and "susceptible" animals, offering increased chances of more effective contacts per unit of time. The number of pigs per pen was another risk factor, already reported for Salmonella $s p$. in breeding pigs by Nollet in 2005 [32]. As in the case of the size of the herd, the greater the number of pigs in the pen, the easier the transmission of infection between pigs, if there are infected pigs in that pen. Interestingly, these two factors were not found significant for "other serotypes" which suggests that "serotype Typhimurium category" could be more associated with transmission between animals than other categories. A protective association, relating to pens without gilts was found. A similar association was also found in the European Union Baseline survey on breeding pigs for maiden gilts [32]. One reason may be that older sows have higher immunity status to Salmonella Typhimurium and may be less susceptible to stress than younger sows although they could be carriers (the test used was pooled faecal culture so it could not detect carriers if they are not shedding) [33]. The last significant risk factor found in this category of the outcome was the boar from another herd which however, has a wide credible interval. A combination of the high odds ratio with a relatively small number of pen faecal samples in this variable category indicates that this association should be a matter of further study. Interestingly, for rodent control, a strong protective effect was noticed towards the Typhimurim group (noticeable by the $\mathrm{OR}=0.11$ ) although not statistically significant. However, it is our opinion that rodent control should not be disregarded from the list of risk factors for $S$. Typhimurim. 


\section{Risk factors for infection with other serotypes of Salmonella}

Concerning the category "other serotypes", the region of the herd was found relevant: samples from herds in the North and Centre Region had higher odds of being positive than samples from herds in the Alentejo Region. Possibilities to explain this finding are that herds in the Centre and North regions are close together or share common management factors. This variable needs further studying to understand whether there are differences in management procedures that were not evaluated by this questionnaire, as this variable did not influence the results of the other variables when it entered the model. Using semen from own boar is a risk factor when compared to using semen from insemination centres only, where the animals are tested and if Salmonella-positive culled. This association has not been reported yet in the literature, probably because in the majority of the countries the semen comes from insemination centres. Pens where pigs feed is not exclusively home produced were at higher risk: the risk is linked to exotic serotypes such as the ones that are isolated in commercial feed; similar association was also found in other studies but for Salmonella sp. [15,32]. There is a protective effect for farrowing pens when compared to mating pens. This can be justified by the hormonal changes in the sow at mating which is similar to the results found in a longitudinal study for sows seven days after weaning [33] where more Salmonella was detected at mating than in the others sector of breeding sows. In that study, this was attributed to hormonal changes that takes place in the sows resulting in follicular growth, ovulation and oestrus behaviour, and also to rise in adrenocorticotrope hormone due to stress. So it was concluded that with stress sows are more susceptible to infection and also carrier sows are more likely to start shedding the pathogen [33]. The control of rodents was considered a protective factor for the presence of "other serotypes": the role of rodents in the transmission of this agent was also highlighted in other studies $[6,8]$. Since rodents could lead to the dissemination of the agent in the herd as a vector that transmits the infection between closed sectors their role must not be underestimated in a control programme. As already mentioned this variable appears as a protective factor to the group Typhimurium or 1,4,5,12:i:- although not statistically significant. This intriguing finding does not compromise the hypothesis of the importance from pig to pig transmission - direct or indirect - in the case of Typhimurium.

\section{Application in control}

The results from this work should be taken into account when implementing control and biosecurity programmes to Salmonella sp., since they highlight the importance to pre-define herd infection status regarding $S$. Typhimurium, and of making a risk profile based on the management practices in place before the adoption of control measures. Control measures should be adapted to suite the type of infection present bearing in mind that for serotype Typhimurium the control of animal source risk factors should be considered, whereas for the other serotypes is it the environmental source risk control that is important.

\section{Conclusion}

In Portugal, the prevalence of herds with breeding pigs that had at least one sample positive to serotype Typhimurium or $S$. Typhimurium-like strains with the antigenic formula: $1,4,5,12: \mathrm{i}$ : was $13.8 \%$ and for the other serotypes $31.7 \%$. A flexible and innovative statistical modelling approach was successfully used here. This provides a framework for similar studies of other diseases as it is straightforward to implement and can be easily generalized. The risk factors for serotype Typhimurium suggest a contagious pattern and the risk factors for other serotypes appeal to be related to environmental factors. The role of rodent control in serotype Typhimurium needs further studies. This study provided valuable information that can be incorporated in future control programmes for Salmonella sp. in breeding pigs in Portugal and other countries.

\section{Additional files}

Additional file 1: Model framework.

Additional file 2: WinBUGS code for the categorical multilevel model.

\section{Competing interests}

The authors do not have any competing interest.

\section{Authors' contributions}

CCG was involved in the design and performed the statistical modelling analysis and drafted the manuscript. TE performed part of the statistical analysis and was involved in the revision of the manuscript for intellectual contents. DM was involved in the revision of the manuscript for intellectual contents and statistical analysis. MVP was involved in the revision of the manuscript for intellectual contents. JNR was involved in the design of the statistical analysis, in the drafting and revision of the manuscript for intellectual content. All authors approved the final manuscript.

\section{Acknowledgments}

We would like to thank FCT for the PhD scholarship (SFRH/BD/40932/2007) and for the strategic research project Pest-OE/AGR/UIO772/2011. We would like also to thank the Portuguese official veterinary authority (DGAV) for the data.

\section{Author details}

${ }^{1}$ Instituto de Ciências Biomédicas Abel Salazar - Universidade do Porto (ICBAS-UP), Population Studies Department, Largo Prof. Abel Salazar, Porto 2, 4099-003, PORTUGAL. ${ }^{2}$ Instituto de Saúde Pública da Universidade do Porto (ISPUP), Rua das Taipas, Porto 135, 4050-600, PORTUGAL. ${ }^{3}$ CEMS, University of Exeter, Harrison Building, North Park Road, Exeter EX4 4QF, UK. ${ }^{4}$ UTAD, 
Veterinary Science Department, Apartado, Vila Real 1013, 5001-801, PORTUGAL.

Received: 16 April 2012 Accepted: 1 November 2012 Published: 21 November 2012

\section{References}

1. EFSA: The community summary report on trends and sources of Zoonoses and zoonotic agents and food-borne outbreaks in the European union 2008. The EFSA Journal 2010, 1496:288.

2. Hald T, Vose D, Wegener HC, Koupeev T: A Bayesian approach to quantify the contribution of animal-food sources to human salmonellosis. Risk Anal 2004, 24(1):255-269.

3. EFSA: Analysis of the baseline survey on the prevalence of Salmonella in holdings with breeding pigs, in the EU, 2008, Part A: Salmonella prevalence estimates. EFSA Journal 2009, 7(12):1377.

4. EFSA: Opinion of the scientific panel on biological hazards on "risk assessment and mitigation options of Salmonella in pig production". The EFSA Journal 2006, 341:1-131

5. Baptista FM, Alban L, Nielsen LR, Domingos I, Pomba C, Almeida V: Use of herd information for predicting salmonella status in Pig herds. Zoonoses Public Health 2010, 57:49-59.

6. Skov MN, Madsen JJ, Rahbek C, Lodal J, Jespersen JB, Jørgensen JC, Dietz $\mathrm{HH}$, Chriél M, Baggesen DL: Transmission of Salmonella between wildlife and meat-production animals in Denmark. J Appl Microbio/ 2008, 105(5):1558-1568

7. Letellier A, Messier S, Paré J, Ménard J, Quessy S: Distribution of Salmonella in swine herds in Québec. Vet Microbiol 1999, 67(4):299-306.

8. Meerburg BG, Kijlstra A: Role of rodents in transmission of Salmonella and Campylobacter. J Sci Food Agric 2007, 87(15):2774-2781.

9. Lo Fo Wong DM, Dahl J, Stege H, Van der Wolf PJ, Leontides L, Von Altrock A, Thorberg BM: Herd-level risk factors for subclinical Salmonella infection in European finishing-pig herds. Prev Vet Med 2004, 62(4):253-266.

10. Poljak Z, Dewey CE, Friendship RM, Martin SW, Christensen J: Multilevel analysis of risk factors for Salmonella shedding in Ontario finishing pigs. Epidemiol Infect 2008, 136(10):1388-1400.

11. Funk J, Gebreyes WA: Risk factors associated with Salmonella prevalence on swine farms. Journal of Swine Health and Production 2004, 12(5):246-251

12. Nollet N, Maes D, De Zutter L, Duchateau L, Houf K, Huysmans K, Imberechts H, Geers R, de Kruif A, Van Hoof J: Risk factors for the herdlevel bacteriologic prevalence of Salmonella in Belgian slaughter pigs. Prev Vet Med 2004, 65(1-2):63-75.

13. Rossel R, Rouillier J, Beloeil PA, Chauvin C, Basta F, Crabos JP, Theau-Audin S: Salmonella en élevage de porcs du Sud-Ouest de la France: séroprévalence en fin d'engraissement et facteurs de risque associés. Journées de la Recherche Porcine 2006, 38:371-378.

14. Bahnson PB, Fedorka-Cray PJ, Ladely SR, Mateus-Pinilla NE: Herd-level risk factors for Salmonella enterica subsp. enterica in U.S. market pigs. Prev Vet Med 2006, 76(3-4):249-262.

15. Benschop J, Stevenson MA, Dahl J, French NP: Towards incorporating spatial risk analysis for Salmonella sero-positivity into the Danish swine surveillance programme. Prev Vet Med 2008, 83(3-4):347-359.

16. Beloeil PA, Chauvin C, Proux K, Fablet C, Madec F, Alioum A: Risk factors for Salmonella seroconversion of fattening pigs in farrow-to-finish herds. Vet Res 2007, 38(6):835-848.

17. Funk J, Wittum TE, LeJeune JT, Rajala-Schultz PJ, Bowman A, Mack A: Evaluation of stocking density and subtherapeutic chlortetracycline on Salmonella enterica subsp enterica shedding in growing swine. Vet Microbiol 2007, 124:202-208.

18. van der Wolf PJ, Wolbers WB, Elbers ARW, van der Heijden HMJF, Koppen JMCC, Hunneman WA, Van Schie FW, Tielen MJM: Herd level husbandry factors associated with the serological Salmonella prevalence in finishing pig herds in The Netherlands. Vet Microbiol 2001, 78(3):205-219.

19. Beloeil PA, Fravalo P, Fablet C, Jolly JP, Eveno E, Hascoet $Y$, Chauvin C, Salvat G, Madec F: Risk factors for Salmonella enterica subsp. enterica shedding by market-age pigs in French farrow-to-finish herds. Prev Vet Med 2004, 63(1-2):103-120

20. Farzan A, Friendship RM, Dewey CE, Poppe C, Funk J: Evaluation of the risk factors for shedding salmonella with or without antimicrobial resistance in swine using multinomial regression method. Zoonoses Public Health 2010, 57:85-93.

21. Anonymous: Report of the task force on Zoonoses data collection on a proposal for technical specifications for a baseline survey on the prevalence of Salmonella in breeding pigs. The EFSA Journal 2007, 99:1-28.

22. Hoorfar J, Mortensen AV: Improved culture methods for isolation of Salmonella organisms from swine feces. Am J Vet Res 2000, 61(11):1426-1429.

23. Arnold ME, Cook A, Davies R: A modelling approach to estimate the sensitivity of pooled faecal samples for isolation of Salmonella in pigs. J R Soc Interface 2005, 2(4):365-372.

24. Gelman A, Rubin DB: Inference from iterative simulation using multiple sequences. Stat Sci 1992, 7:457-511.

25. Kostoulas P, Leontides L, Enøe C, Billinis C, Florou M, Sofia M: Bayesian estimation of sensitivity and specificity of serum ELISA and faecal culture for diagnosis of paratuberculosis in Greek dairy sheep and goats. Prev Vet Med 2006, 76(1-2):56-73.

26. Best NG, Cowles MK, Vines SK: CODA Manual Version 0.30. Cambridge, UK: MCR Biostatistics Unit; 1995.

27. Congdon PD (Ed): Applied Bayesian hierarchical methods. Boca Raton: Chapman and hall/ CRC; 2010.

28. Gilks WR, Richardson S, Spiegelhalter DJ: Markov chain Monte Carlo in practice. Boca Raton: Chapman and Hall/CRC; 1996.

29. Alban L, Olsen AM, Nielsen B, Sorensen R, Jessen B: Qualitative and quantitative risk assessment for human salmonellosis due to multi-resistant salmonella typhimurium DT104 from consumption of Danish dry-cured pork sausages. Prev Vet Med 2002, 52(3-4):251-265.

30. (BIOHAZ) EPoBH: Scientific opinion on monitoring and assessment of the public health risk of "Salmonella Typhimurium-like" strains. EFSA Journal 2010, 8(10):48

31. Arnold ME, Cook AJ: Estimation of sample sizes for pooled faecal sampling for detection of Salmonella in pigs. Epidemiol Infect 2009, 137(12):1734-1741.

32. EFSA: Analysis of the baseline survey on the prevalence of Salmonella in holdings with breeding pigs, in the EU, 2008 Part B: factors associated with Salmonella pen positivity. The EFSA Journal 2011, 9(7):2329.

33. Nollet N, Houf K, Dewulf J, De Kruif A, De Zutter L, Maes D: Salmonella in sows: a longitudinal study in farrow-to-finish pig herds. Vet Res 2005, 36(4):645-656.

doi:10.1186/1746-6148-8-226

Cite this article as: Correia-Gomes et al:: Assessing risk profiles for Salmonella serotypes in breeding pig operations in Portugal using a Bayesian hierarchical model. BMC Veterinary Research 2012 8:226.

\section{Submit your next manuscript to BioMed Central and take full advantage of:}

- Convenient online submission

- Thorough peer review

- No space constraints or color figure charges

- Immediate publication on acceptance

- Inclusion in PubMed, CAS, Scopus and Google Scholar

- Research which is freely available for redistribution 